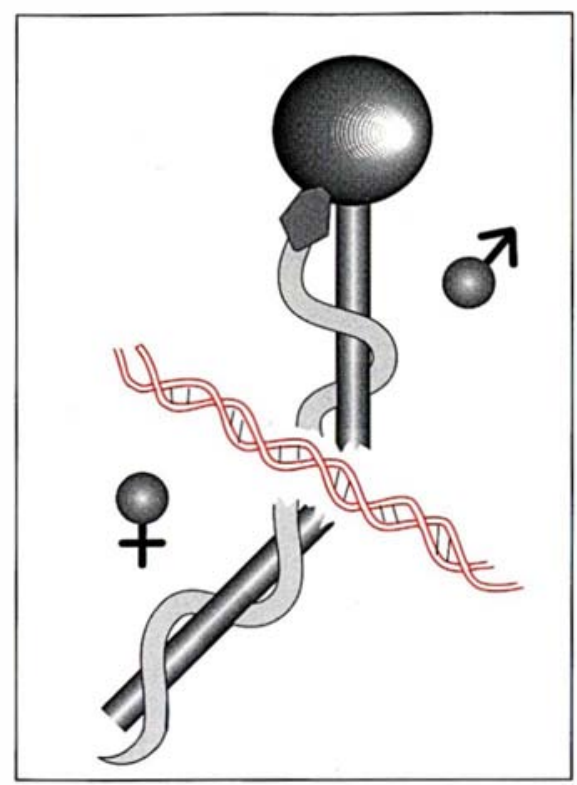

Préambule

Comité consultatif national d'éthique pour les sciences de la vic et de la santé, 101. rue de Tolbiac. 756.5.t Paris Cedex 13

\section{Application des tests génétiques aux études individuelles, études familiales et études de populations (problèmes des "banques" d'ADN, des "banques" de cellules et de l'informatisation des données)}

\section{$\mathbf{L}$} es progrès de la génétique moléculaire ont ouvert un immense champ d'investigation des caractères génétiques individuels. Comme dans toute recherche, on doit distinguer l'acquisition des connaissances qui ne pose pas de problèmes éthiques nouveaux, et l'utilisation des connaissances acquises qui conduit à mettre en balance les conséquences bénéfiques pour l'individu et la Santé Publique, et les éventuels effets pervers à ces deux niveaux.

Dans la pratique, il est bien difficile d'établir des séparations nettes et il $\mathrm{y}$ a toujours intrication et superposition au moins partielles.

\section{Le champ d'application}

1. Les maladies dont le mode de transmission génétique est connu

Dans le domaine des maladies monogéniques, l'analyse de l'ADN par biologie moléculaire a bouleversé ce domaine de la pathologie. Ces progrès ont permis

- tout d'abord la localisation précise du gène responsable sur un segment du chromosome, c'est la cartographie génétique ;

- puis, dans quelques cas, l'isolement et la caractérisation du gène, et ensuite l'identification de la protéine (myopathie, mucoviscidose).
La localisation des gènes responsables de ces maladies (et par la suite l'identification du gène) repose sur de vastes études familiales.

La technique d'analyse est le " marquage " du segment de chromosome porteur du gène muté grâce à une liaison étroite avec des polymorphismes de l'ADN. On ignore le plus souvent la signification de ces caractères polymorphes, mais situés à proximité du gène et si possible de part et d'autre, ils permettent, dans une famille, de suivre la transmission du gène. Les différentes formes de polymorphismes sont indépendantes du gène, ce qui fait que dans une famille, le gène muté se trouvera " lié " à la forme "A " d'un marqueur et dans une autre famille à la forme " B ", d'où la nécessité d'étudier de manière extensive chaque famille sur plusieurs générations.

Après les recherches sur la localisation du gène, cette analyse de liaison avec un polymorphisme est utilisée quotidiennement pour les diagnostics demandés par les familles, pour connaître les sujets porteurs-sains du gène muté (en particulier dans les maladies liées au sexe) et pour éventuellement permettre un diagnostic prénatal. En outre, la constitution des banques d'ADN ou de banques de cellules de sujets atteints permet, après leur décès, d'offrir aux familles des possibilités de diagnostic; c'est ce qui a été réalisé pour des maladies comme la myopathie ou 
la mucoviscidose et de nombreuses maladies génétiques.

Ces études génétiques familiales et ces banques sont donc indispensables pour les diagnostics même lorsque le gène est connu; la même maladie peut résulter d'un grand nombre de mutations et il faut connaître les mutations dans chaque famille pour leur offrir des possibilités de diagnostic.

Il faut penser que ces informations devraient être conservées et disponibles pendant un temps long (au moins 20 ans) pour assurer les possibilités de diagnostic aux générations suivantes. Ces informations familiales comporteront en outre des renseignements sur les filiations (légitimes ou illégitimes) qui devront rester secrets.

2. Les recherches sur des facteurs génétiques pouvant jouer un rôle dans les maladies non monogéniques: maladies multifactorielles

Ces recherches sont surtout orientées vers les maladies neuro-psychiatriques (schizophrénie, psychose maniacodépressive, maladie d'Alzheimer...), les cancers, les maladies cardiovasculaires, les maladies autoimmunes. Les recherches tentent de découvrir des facteurs moléculaires de risque génétique, de les localiser et de les identifier. Grâce à ces "gènes de susceptibilité ", on pourrait déterminer des groupes de sujets à risque accru et, dans ces groupes, mettre en évidence d'autres facteurs favorisants (génétiques, environnementaux...). Une conduite préventive pourrait, après une appréciation de sa valeur, être éventuellement envisagée.

A l'inverse de la situation dans les maladies monogéniques où c'est un diagnostic de certitude d'un gène muté, même si l'expression clinique de la maladie peut varier, dans les maladies multifactorielles, il s'agit toujours d'un diagnostic de probabilité.

Dans le domaine du cancer, on connaît probablement pour tous les types de cancer des formes héréditaires, qui sont fréquentes pour certains cancers (rétinoblastome, par exemple) mais rares pour d'autres. Des gènes de susceptibilité à divers cancers ont déjà été localisés. Grâce à ces gènes $\mathrm{m} / \mathrm{s} n^{\circ} 8$, vol. 7 , octobre 91 de susceptibilité le dépistage de sujets à risque pourrait permettre soit de prévenir l'apparition de ces tumeurs si des facteurs environnementaux favorisants sont connus, soit de déceler plus précocement les tumeurs en sélectionnant des groupes à risque accru et ainsi instituer une attitude thérapeutique, soit après la découverte de la tumeur apporter des éléments pronostiques conduisant la thérapeutique.

Dans le domaine neuropsychiatrique, la situation est et demeurera plus complexe : la nosologie reste soumise à des révisions fréquentes, la pénétrance génétique est incertaine, l'étude du rapport entre facteurs liés aux gènes et à l'environnement humain souffre de lacunes méthodologiques, et les retombées de toute recherche, tant sur l'environnement que sur le sujet, sont spécialement lourdes.

Pour toutes ces recherches, on envisage de larges études familiales avec constitution de banques d'ADN et de banques de cellules. Pour de nombreuses maladies multifactorielles, il est douteux que la stratégie fondée sur la localisation de gènes grâce à des marqueurs génétiques polymorphes qui a été couronnée de succès pour les maladies monogéniques, soit la seule stratégie. Dans ce domaine, il s'agit de recherches importantes pour l'acquisition des connaissances mais, dans l'optique d'une application, le "bénéfice " éventuel pour l'individu, les familles et la Santé Publique est à long terme et aléatoire.

\section{Tests d'identification génique}

Les polymorphismes de l'ADN peuvent être utilisés comme test d'identification génétique (ce sont les techniques d'empreinte génétique, $D N A$ fingerprint) (voir l'avis du CCNE du 19 décembre 1989).

Leur utilisation dans le domaine pénal (identification de cadavre, viol...) demande la connaissance de la répartition de ces caractères polymorphes dans la population. Par exemple, un caractère polymorphe rare dans une région peut être fréquent dans un autre. Les services scientifiques associés à la police souhaitent donc connaître la répartition de ces caractères polymorphes dans des populations particulières (c'est ce qui s'est passé à New York). Dans notre pays s'il est assez facile d'avoir une idée de la répartition de ces caractères dans les populations régionales, le problème des territoires d'outre-mer (Antilles, par exemple) ou des groupes d'immigrants (Maghreb...) est beaucoup plus délicat.

Problèmes et orientations éthiques Les problèmes éthiques concernent deux objets connexes mais différents : - le recueil, l'utilisation, la conservation et la diffusion de l'information génétique d'un individu obtenue par les tests;

- le recueil, la conservation, la transmission et l'utilisation à terme de l'ADN, élément du corps humain et support d'informations génétiques.

Certaines exigences éthiques devraient être respectées lors de ces diverses opérations.

\section{- Le respect de l'autonomie}

Il y a lieu de respecter le droit de l'individu à prendre une décision "informée " sur la pratique d'un examen qui pourrait révéler la présence d'un gène muté ou de susceptibilité dans son capital génétique et, quelqu'en soit le résultat, pourrait avoir un effet profond sur sa vie.

Pour ce faire, trois conditions sont essentielles :

- une liberté de choix en dehors de toute coercition ;

- une compréhension complète des indications d'une telle décision;

- le sujet demandeur doit avoir la capacité juridique de donner un consentement éclairé.

Cette exigence d'autonomie s'appliquera non seulement au sujet de qui émane la première demande dans une famille à risque, mais aussi à tous les autres membres de la famille, qu'ils soient potentiellement à risque ou non, et ceci sur plusieurs générations.

La constitution de ces banques d'ADN posera différents types de problèmes.

Au moment du prélèvement de sang à l'ensemble d'une famille, comment sera faite l'information à chacun de ses membres, quel type de pression fera-t-on pour obtenir du sang de 
sujets réticents ? Le sujet demandeur doit contacter lui-même les membres de sa famille, cette demande pouvant être longue et délicate.

Il est difficilement concevable que des personnes ignorant l'objet de l'étude génétique, éventuellement même la nature précise de l'affection dont est atteint leur parent, puissent être sollicités directement par un médecin qui aurait obtenu du malade ou de ses parents leurs noms et adresses. Pour les maladies où les possibilités de diagnostic existent, c'est en général la nécessité d'assurer un diagnostic prénatal à une des femmes enceintes qui motive l'ensemble de la famille.

Qu'en sera-t-il lorsqu'il s'agira seulement d'un programme de recherches? Dans les maladies neuro-psychiatriques, le patient peut ne pas jouir d'un discernement suffisant pour appréhender le problème pour luimême et il peut répandre dans sa famille un message involontairement altéré. Il appartient alors au médecin-psychiatre d'informer soigneusement et directement chaque personne pressentie.

Comment justifier le prélèvement chez des mineurs dans un seul but de recherche?

\section{- Le droit de savoir}

Il faut tenir chaque sujet au courant des éventuels résultats et l'informer clairement de leur signification.

Mais la connaissance peut limiter l'autonomie de l'individu.

Ce peut être la situation dans les maladies monogéniques où la connaissance peut modifier le comportement d'un sujet, par exemple dans les maladies liées au sexe, connaissance chez une femme d'être conductrice de la maladie (myopathie, hémophilie...); dans les maladies dominantes, connaissance d'être porteur du gène muté et donc d'être atteint dans l'avenir (chorée de Huntington). Il peut donc refuser d'avoir connaissance des résultats.

Plus complexe pourra être la connaissance d'une probabilité, comme c'est le cas des gènes de "susceptibilité ". Un sujet chez lequel est mis en évidence un gène de susceptibilité à un cancer ou à une maladie neuropsychiatrique, sans autre conséquence transformer - éventuellement et après combien de temps ? - la probabilité en certitude, sera-t-il autonome ? On peut se poser la question de l'opportunité de communiquer les résultats d'un caractère du génome qui conduirait seulement à une évaluation probabiliste d'un risque d'une affection grave sans qu'aucune conduite préventive efficace puisse être conseillée et entreprise.

Peut-on dire à un enfant son "destin biologique"?

"Le hiatus entre les possibilités de prédire, qui augmentent rapidement, et les possibilités d'attitudes préventives et curatives s'élargit avec l'impatience de la société d'utiliser prématurément les résultats des recherches, soulevant ainsi d'importantes questions éthiques" (Éric Lander).

"On ne peut pas affirmer qu'à n'importe quel progrès dans la recherche biologique doit correspondre un élargissement du "droit de savoir" des particuliers et des institutions publiques ou privées. Il faut trouver un point d'équilibre entre les bénéfices qui peuvent être apportés par l'analyse de l'ADN et d'autres valeurs d'ordre juridique et social " (Stefano Rodota).

- La confidentialité et le respect de la vie privée

Le secret médical doit être respecté non seulement vis-à-vis de tiers, mais aussi vis-à-vis des autres membres de la famille.

Toute détermination de caractères de génotype d'un individu ne devrait être entreprise que s'il a spécifiquement donné son autorisation. On peut se poser la question de l'extension des recherches à des caractéristiques dans un domaine qui est étranger à celui pour lequel l'autorisation a été donnée au moment du prélèvement (du fait des possibilités de conservation de l'ADN par exemple).

Aucune information sur le génotype d'un individu ne devrait être donnée sans son consentement formel.

Les études familiales peuvent mettre en évidence la nature extraconjugale de filiation qui est méconnue ou cachée, mais dont la connaissance sur le plan biologique est indispensable pour l'interprétation des résultats et éventuellement pour leur utilisation dans un but diagnostique.

Ces informations peuvent avoir des effets bénéfiques pour l'individu pour sa santé, pour ses projets familiaux. Elles peuvent aussi avoir des effets pervers. Il va se poser le problème de l'utilisation de ces informations à l'embauche, dans les assurances et dans toute autre institution qui a un intérêt organisationnel ou financier d'éviter des risques.

A côté de l'intérêt de protéger certains sujets à un risque lié à une activité professionnelle (sensibilité à une substance toxique par exemple), il y a danger de marginaliser des groupes de sujets du fait de risques généraux de santé.

D'une manière plus générale, va se poser le problème de la gestion de ces données génétiques et de leur informatisation en particulier dans les "banques d'ADN ". Cette gestion est indispensable car les renseignements obtenus dans ces familles doivent pouvoir servir éventuellement aux générations futures de ces familles (par exemple pour les enfants qui sont nés grâce aux diagnostics).

\section{L'information et la formation des praticiens}

Toutes ces règles de conduite reposent en pratique sur l'information et sa rigueur, et par voie de conséquence sur une bonne formation des praticiens dans ce domaine. Or, le caractère récent des applications des recherches fondamentales en génétique fait que les praticiens n'ont généralement pas reçu cet enseignement pendant leurs études.

La génétique médicale, clinique et biologique, n'est pas encore une spécialité reconnue et structurée. Il n'y a pas de cursus d'enseignement spécialisé, d'internat qualifiant, et la génétique n'est pas officiellement une spécialité médicale.

Devant la demande qui s'accroît et la nécessité d'une information de qualité, si on veut éviter des dérives il est important que cet aspect pratique de l'application soit envisagé avec autant de sérieux et de moyens qu'est mise en œuvre la recherche fondamentale en génétique 


\section{Avis du Comité Consultatif National d'Éthique}

Les tests génétiques

Les progrès de la génétique humaine permettent l'investigation des caractères génétiques individuels sur un seul échantillon d'ADN.

Ces techniques ont déjà conduit à des avancées spectaculaires dans l'acquisition des connaissances et les programmes de recherches en cours devraient encore accélérer ces progrès. L'application des connaissances ainsi acquises apporte dé jà des possibilités de diagnostic dans des maladies génétiques graves

Mais ces applications conduisent à mettre en balance leurs conséquences bénéfiques pour l'individu et pour la Santé Publique et leurs éventuels effets pervers à ces deux niveaux. En pratique, il est bien difficile d'établir une frontière nette entre recherche et application, il y a toujours intrication et superposition au moins partielle.

Dans ce domaine des règles éthiques sont indispensables.

\section{- Au niveau individuel}

Le génome d'un individu, parce qu'il relève de son être plutôt que de son avoir, ne peut pas être l'objet de commerce, tout comme les autres composants physiques de sa personne.

Cette "non-propriété " ne s'oppose pas à une recherche ou analyse des éléments constituant le génome.

Cette analyse doit présenter une utilité évidente pour celui qui s'y soumet ou sa famille, ou doit participer à un programme de recherches jugé utile par la Communauté scientifique.

- Le prélèvement pour fins d'analyse de l'ADN doit demeurer un acte médical et ne relever que d'une indication médicalement reconnue.

- Toute détermination de caractères du génome d'un individu ne devra être entreprise qu'après une information directe sur les analyses prévues et qu'après s'être assuré de la compréhension complète des implications éventuelles de la connaissance des résultats, avec un délai de réflexion. Le consentement du sujet est donné pour des analyses spécifiques. L'extension des investigations à des caractéristiques du génome dans un domaine étranger à celui pour lequel le consen- tement a été donné au moment du prélèvement devra faire l'objet d'une nouvelle information et d'un nouveau consentement. Il est possible de demander le retrait du prélèvement. - Chaque individu doit en principe être tenu au courant des éventuels résultats des investigations et être informé clairement de leur signification. Mais comme ces résultats, qu'il s'agisse de diagnostics de certitude ou de diagnostics de probabilité, peuvent avoir des conséquences sur la vie et le comportement de l'individu ayant compris la portée éventuelle de ces analyses sur sa vie, chacun peut dès le moment du prélèvement demander à ne pas être informé des résultats. Pour certaines recherches fondamentales, en particulier celles cherchant à déterminer des facteurs de risque dans des maladies multifactorielles où aucune attitude préventive n'est sérieusement envisageable, il serait préférable de préciser avant le prélèvement qu'aucun résultat, quel qu'il soit, ne sera communiqué.

- Aucun résultat sur les caractères du génome d'un individu ne doit être communiqué à ses parents, à des tiers et à tout organisme public ou privé sans son consentement formel. La communication des résultats dans le cadre d'un diagnostic médical devra se faire par l'intermédiaire d'un médecin pouvant donner toute l'information sur leur signification.

- L'individu dont le génome devrait être analysé doit avoir la capacité juridique de donner un consentement et doit pouvoir comprendre la signification des résultats.

Dans le cas contraire (mineurs...) les examens devraient être réservés à des situations exceptionnelles où la connaissance de caractères du génome est indispensable à l'analyse de la transmission d'un gène muté responsable d'une maladie monogénique répondant à la demande d'un diagnostic dans la famille.

- Les parents peuvent demander l'analyse d'un génotype de leur enfant seulement si la maladie liée à ce génotype peut se déclarer avant 18 ans ou peut bénéficier de mesures préventives instaurées avant 18 ans. - Une femme enceinte avec un risque de maladie monogénique grave peut demander le diagnostic prénatal du génotype de l'enfant qu'elle attend.
- Un prélèvement chez un mineur dans un seul but de recherche ne doit être envisagé qu'après a voir soumis le protocole à un Comité d'Éthique.

\section{- Les études familiales}

Les études familiales du génotype sont indispensables, soit pour la recherche d'acquisition des connaissances en génétique, soit pour les applications dans des buts diagnostiques. Il peut ne pas y avoir coïncidence entre la famille biologique et la famille légale et toute divulgation est à rejeter. La connaissance de caractéristiques génétiques n'appartient pas collectivement à la famille biologique.

- Toutes les règles énumérées au niveau individuel doivent être respectées et s'appliquer à tous les membres de la famille, qu'ils soient potentiellement à risque ou non, la qualité de l'information représentant une des règles les plus importantes avant tout prélèvement.

Les méthodes d'accès aux différents membres d'une famille doivent respecter certaines règles :

- le sujet demandeur devra contacter lui-même les membres de sa famille, éventuellement assisté par un médecin car il peut lui être difficile d'expliquer techniquement la demande ; - il est difficilement concevable que des personnes ignorant la nature de l'affection dont est atteint un parent et l'objet de l'étude génétique puissent être sollicités directement à partir de leurs coordonnées transmises par un parent. Dans les maladies neuropsychiatriques, le sujet peut ne pas jouir d'un discernement suffisant pour appréhender le problème pour luimême et peut répandre dans sa famille un message involontairement altéré. Il appartient au médecin d'informer soigneusement et directement chaque personne pressentie.

Les mêmes difficultés peuvent se poser si c'est un mineur qui est le point de départ de l'étude familiale. L'accès aux membres de la famille devra se faire par l'intermédiaire de ses parents.

- Le qualité de l'information et la formation des praticiens

La qualité de l'information est le support indispensable de toutes les applications de tests génétiques.

Il faut insister sur le caractère urgent d'assurer la formation des praticiens 
dans ce domaine par l'enseignement de la génétique médicale et l'organisation pratique de cette activité médicale non encore reconnue comme spécialité.

- Les registres, les banques d'ADN, l'informatisation

Les banques d'ADN impliquent nécessairement l'informatisation de données nominatives concernant les différentes personnes ayant fait l'objet de prélèvements de cellules conservées dans la banque. De ce fait, toutes les règles définies pour la tenue et l'utilisation des registres médicaux sont applicables conjointement à ces banques elles-mêmes et aux registres qui en sont le complément nécessaire.

Ces règles ont été définies dans l'Avis de principe, émis par le Comité le 6 mars 1985, ainsi que dans divers autres Avis qui ont précisé et complété celui-ci. Elles sont les suivantes.

1. La tenue des registres devrait être réservée à un petit nombre de centres agréés par une autorité publique et présentant toutes les garanties nécessaires sur le plan scientifique et éthique. Le même principe devrait valoir pour les banques d'ADN.

2. Les exigences du secret médical impliquent que le centre tenant un registre soit placé sous l'autorité d'un médecin et que celui-ci accepte de se considérer, à l'égard des praticiens lui transmettant les informations enregistrées, comme un consultant, lié, bien entendu, par le secret médical, et devant faire connaître aux praticiens en cause son sentiment sur le diagnostic et la thérapeutique qu'appelle le cas signalé.

3. La loi du 6 janvier 1978 sur l'Informatique, les Fichiers et les Libertés implique que toute personne intéressée :

a. dûment avertie de la faculté qu'elle a de s'opposer à la transmission des renseignements (donc aussi de l'ADN prélevé) la concernant n'ait pas manifesté d'opposition et, en pratique, ait donné un accord écrit à cette transmission ;

b. ait accès à tout moment, par l'intermédiaire d'un médecin de son choix, aux renseignements enregistrés ;

c. soit avertie de son droit de demander, si elle a un motif sérieux de le faire, la suppression du registre des renseignements la concernant, donc le retrait de la banque de son ADN.

4. Le Comité ne s'estime pas qualifié pour se prononcer sur la question de savoir si le prélèvement et la transmission de cellules humaines à une banque d'ADN, et l'insertion corrélative de renseignements nominatifs dans un registre, entre dans le champ d'application de la Loi du 20 décembre 1988, modifiée le 23 janvier 1990, laquelle exige qu'avant d'entreprendre une recherche sur l'être humain, tout investigateur soumette le projet au Comité Consultatif de Protection des Personnes dans la recherche biomédicale, ayant son siège dans la région où l'investigateur exerce son activité.

5. Il devrait être interdit à tout tiers, notamment employeur ou entreprise d'assurances, non seulement d'avoir accès aux renseignements contenus dans un registre, donc par une banque d'ADN, ce qui est déjà impliqué par les règles précédentes, mais aussi de demander aux intéressés euxmêmes la production des éléments d'information les concernant dont dispose le registre ou la banque d'ADN. 6. La recherche des empreintes génétiques et leur utilisation devraient être subordonnées à l'autorisation préalable d'une juridiction, appréciant si cette recherche ou utilisation est justifiée en droit et en fait.

Il est souhaitable que le législateur, seul en mesure de donner leur entier effet à certaines, au moins, des garanties ainsi énumérées, adopte les mesures nécessaires à cette fin 